\title{
EXPERIENCIA DE TRATAMIENTO PALIATIVO EN METÁSTASIS ÓSEAS*
}

\author{
Drs. Lorena Vargas G. ${ }^{1,2}$, Rodrigo Barrientos G. ${ }^{1,2}$, Ramón Baeza B. ${ }^{1}$ \\ 1 Clínica IRAM. \\ 2 Facultad de Medicina, Universidad Diego Portales. \\ Santiago, Chile.
}

\begin{abstract}
\section{Bone metastases and palliative radiotherapy}

Background: About $40-50 \%$ of cancer patients have indication of palliative radiotherapy during the course of their disease. Bone metastases are the most common cause of cancer-related pain. Evidence shows that there is no statistically significant difference between long and short treatments modalities in pain relief. The aim of this study is to review the experience in treatment of patients requiring palliative radiotherapy for bone metastases treated in Clinica IRAM during 2012. Methods: Descriptive retrospective analysis of medical records of patients treated during 2012 at Clínica IRAM with palliative radiotherapy for painful bone metastases. Results: 197 patients were included. The median time between onset of symptoms and initiation of treatment was 4 months (1-42 months). The scheme of $8 \mathrm{~Gy}$ in one fraction was the most prescribed. Sixty-two percent of patients had a very good/good response to treatment, and $10 \%$ had a poor response. The median survival was 10.5 months. One, 12 and 24 months survival was $91.8 \%, 46.3 \%, 28.7 \%$ respectively. Ten patients were re-irradiated, 7 of them had received $8 \mathrm{~Gy}$ in a single fraction as first treatment dose. Conclusion: The palliative treatment of cancer patients is an important indication of radiotherapy. The most common indication for treatment was $8 \mathrm{~Gy} / 1$ fraction; however, there was a $40 \%$ multi fractionated treatments. Since pain relief is similar between different treatment modalities, to offer a shorter treatment appears to be a more reasonable option.
\end{abstract}

Key words: Metastases, palliative therapy, radiotherapy.

\section{Resumen}

Introducción: Aproximadamente 40-50\% de los pacientes con cáncer tiene indicación de radioterapia paliativa durante el curso de su enfermedad. Las metástasis óseas son la causa más frecuente de dolor relacionado al cáncer. Existe evidencia que demuestra que no existe diferencia estadísticamente significativa entre esquemas de tratamientos prolongados y cortos en alivio del dolor. Objetivo: Revisión de la experiencia en el tratamiento de pacientes con indicación de radioterapia paliativa por metástasis ósea tratados en Clínica

*Recibido el 15 de marzo de 2015 y aceptado para publicación el 28 de agosto de 2015.

Los autores no refieren conflictos de interés.

Este artículo no tuvo apoyo financiero.

Correspondencia: Dra. Lorena Vargas G. lorenavargasg@gmail.com 
IRAM en el año 2012. Método: Análisis retrospectivo descriptivo de fichas clínicas de pacientes tratados el año 2012 en Clínica IRAM con indicación de radioterapia paliativa por metástasis óseas. Resultados: Se incluyeron 197 pacientes. La mediana de tiempo entre inicio de síntomas e inicio de tratamiento fue de 4 meses (1-42 meses). El esquema de 8 Gy en 1 fracción fue el más prescrito; $62 \%$ de los pacientes tuvo una respuesta muy buena/buena al tratamiento, y $10 \%$ tuvo una respuesta pobre. La mediana de sobrevida fue 10,5 meses. La sobrevida al mes, 12 y 24 meses fue 91,8\%, 46,3\%, 28,7\% respectivamente. Diez pacientes fueron reirradiados, 7 de ellos tuvieron primer esquema de monodosis $8 \mathrm{~Gy} / 1$ fracción. Conclusión: El manejo paliativo de pacientes con cáncer representa una importante indicación de radioterapia. La indicación más frecuente de tratamiento fue $8 \mathrm{~Gy} / 1$ fracción, sin embargo, hubo un $40 \%$ de tratamientos multifraccionados. Dado que el alivio del dolor es similar entre distintas modalidades terapéuticas, realizar un tratamiento más corto parece ser una alternativa más razonable.

Palabras clave: Metástasis, tratamiento paliativo, radioterapia.

\section{Introducción}

La radioterapia tiene un importante rol en el manejo del paciente con cáncer avanzado. Aproximadamente un 40 a $50 \%$ de estos pacientes tienen indicación de radioterapia paliativa ${ }^{1,2}$. Las principales indicaciones son alivio del dolor óseo, control de hemorragia y obstrucción. También es importante en el manejo de urgencias oncológicas como síndrome de vena cava superior, compresión medular y aumento de la presión intracraneana por metástasis cerebrales $^{3}$. Las metástasis óseas son la causa más frecuente de dolor relacionado al cáncer ${ }^{4,5}$, presentándose muchas veces como primera evidencia de enfermedad diseminada. Un 50\% de los pacientes con metástasis óseas requerirá radioterapia para el manejo del do${ }{ }^{6}{ }^{6}$. Las metástasis óseas son frecuentes en cáncer de mama $(70 \%)$, próstata $(70 \%)$ y pulmón $(35 \%)^{5}$. Sesenta y cinco a $75 \%$ de los pacientes con metástasis óseas tendrán dolor o compromiso funcional. Además, las metástasis óseas pueden causar otras complicaciones como fracturas patológicas, disminución en producción de médula ósea, compresión medular o nerviosa e hipercalcemia ${ }^{2,5}$. Es por esta razón que ocasionalmente la indicación de radioterapia paliativa es electiva/preventiva, es decir, para disminuir el riesgo de fractura y/o colapso óseo.

La efectividad de esta modalidad terapéutica es alta, es así que hasta el $80 \%$ de los pacientes tratados con radioterapia por metástasis óseas presentan disminución del dolor, y alrededor de un 50\% de ellos alcanza analgesia completa ${ }^{6,7}$.

Se han publicado diversos estudios que demuestran la equivalencia entre diferentes relaciones de dosis y fraccionamiento de la radioterapia ${ }^{4}$. Al comparar planes de alta dosis por fracción usando una fracción solamente con planes de mayor dosis y fraccionamiento, no se aprecian diferencias estadísticamente significativas entre ambas modalidades terapéuticas tanto en el alivio ${ }^{4-6}$ como en la progresión de dolor y duración de la respuesta ${ }^{6}$. Hasta el noventa por ciento de los pacientes con metástasis óseas refiere algún grado de alivio del dolor al recibir un fraccionamiento corto de radioterapia ${ }^{1}$.

Por otro lado, es importante considerar que el tipo de tratamiento debe ser elegido de acuerdo a las condiciones y expectativas de vida de cada paciente. Un estudio que evaluó a pacientes que murieron al mes de haber ingresado a radioterapia, mostró que un $77 \%$ de ellos tenía planes de tratamiento largos de 30 a 40 Gy, sólo un 58\% de los pacientes completaron el plan y la mitad de los pacientes pasó el $60 \%$ de su vida restante en terapia ${ }^{8}$.

Es importante procurar alcanzar el máximo efecto con el mínimo tratamiento en el manejo paliativo de los pacientes con cáncer con expectativa de vida limitada, con el fin de aumentar la calidad de vida restante ${ }^{5}$.

A pesar de la evidencia que muestra que no existen diferencias en la respuesta de estos pacientes a los distintos esquemas, muchos médicos se muestran reticentes a ofrecer tratamientos acortados de radioterapia con solamente una fracción ${ }^{4,6}$.

El objetivo de este trabajo es reportar la experiencia en tratamiento paliativo por metástasis óseas en un grupo de pacientes tratados con radioterapia en Clínica IRAM durante el año 2012.

\section{Materiales y Método}

Se realizó un estudio retrospectivo, descriptivo, con revisión de fichas clínicas de pacientes tratados en Clínica IRAM durante el año 2012 con indicación de radioterapia paliativa por metástasis óseas. Se analizó el tumor primario, tiempo desde inicio de los síntomas hasta inicio de tratamiento, dosis de radioterapia indicada, tolerancia al tratamiento, frecuencia y/o necesidad de re-irradiación.

\section{Resultados}

Se estudiaron 197 pacientes, 131 mujeres, 66 hombres. El tiempo entre inicio de síntomas e inicio 
Tabla 1. Tipo histológico de tumor primario

\begin{tabular}{|c|c|}
\hline Tipo histológico & n $(\%)$ \\
\hline Mama & $100(51)$ \\
\hline Pulmón & $30(15)$ \\
\hline Próstata & $28(14)$ \\
\hline Riñón & 17 (9) \\
\hline Recto & 4 (2) \\
\hline Gástrico & $2(1)$ \\
\hline Hepatocarcinoma & $2(1)$ \\
\hline Digestivo NE* & $2(1)$ \\
\hline Ovario & $2(1)$ \\
\hline Cérvix & $1 \quad(0,5)$ \\
\hline Ewing & $1 \quad(0,5)$ \\
\hline Vejiga & $2(1)$ \\
\hline Sarcoma PB** & $2(1)$ \\
\hline Tiroides & $1(0,5)$ \\
\hline Parótida & $1 \quad(0,5)$ \\
\hline Otro & $2(1)$ \\
\hline
\end{tabular}

*NE: no especificado. **PB: partes blandas.

de radioterapia fue entre 1 a 42 meses, con una mediana de 4 meses.

Los tipos histológicos más frecuentes fueron mama 51\% (100/197), pulmón 15\% (30/197), próstata $14 \%(28 / 197)$ y riñón $9 \%(17 / 197)$ (Tabla 1$)$.

La distribución de los tratamientos indicados se muestra en la Tabla 2.

Diez de los pacientes estudiados recibieron retratamiento, 7 de ellos habían recibido dosis de 8 Gy en 1 fracción en el primer tratamiento, 2 pacientes que habían sido tratados con 20 Gy en 4 fracciones y 1 paciente que recibió inicialmente 30 Gy en 10
Tabla 2. Distribución de dosis de tratamientos indicados

\begin{tabular}{|lr|}
\hline Dosis & n (\%) \\
$8 \mathrm{~Gy} / 1 \mathrm{Fx}$ & $123(60)$ \\
$20 \mathrm{~Gy} / 4 \mathrm{Fx}$ & $37(18)$ \\
$20 \mathrm{~Gy} / 5 \mathrm{Fx}$ & $13(6)$ \\
$25 \mathrm{~Gy} / 5 \mathrm{Fx}$ & $6(3)$ \\
$30 \mathrm{~Gy} / 10 \mathrm{Fx}$ & $22(11)$ \\
$10 \mathrm{~Gy} / 3 \mathrm{Fx}$ & $3(1)$ \\
$30 \mathrm{~Gy} / 5 \mathrm{Fx}$ & $2(1)$ \\
\hline
\end{tabular}

Fx: fracciones.

Tabla 3. Porcentaje de sobrevida (SV) en tiempo

\begin{tabular}{|cc|}
\hline SV (meses) & \% \\
\hline 1 & 92 \\
3 & 78 \\
6 & 62 \\
12 & 46 \\
18 & 34 \\
24 & 29 \\
\hline
\end{tabular}

fracciones.

La mediana de sobrevida fue de 10,5 meses, con una mortalidad de $8,2 \%$ al mes de terminado el tratamiento (Figura 1). A 12, 18 y 24 meses la sobrevida observada fue $46,3 \%, 33,6 \%$ y $28,7 \%$ respectivamente (Tabla 3 ).

Con respecto a la respuesta al tratamiento, medida de acuerdo a la evaluación del médico tratante, $62 \%$ de los pacientes tuvo una respuesta muy buena/ buena, y en el $10 \%$ se obtuvo una respuesta pobre al tratamiento. El resto de los pacientes no fueron evaluados para esta variable.

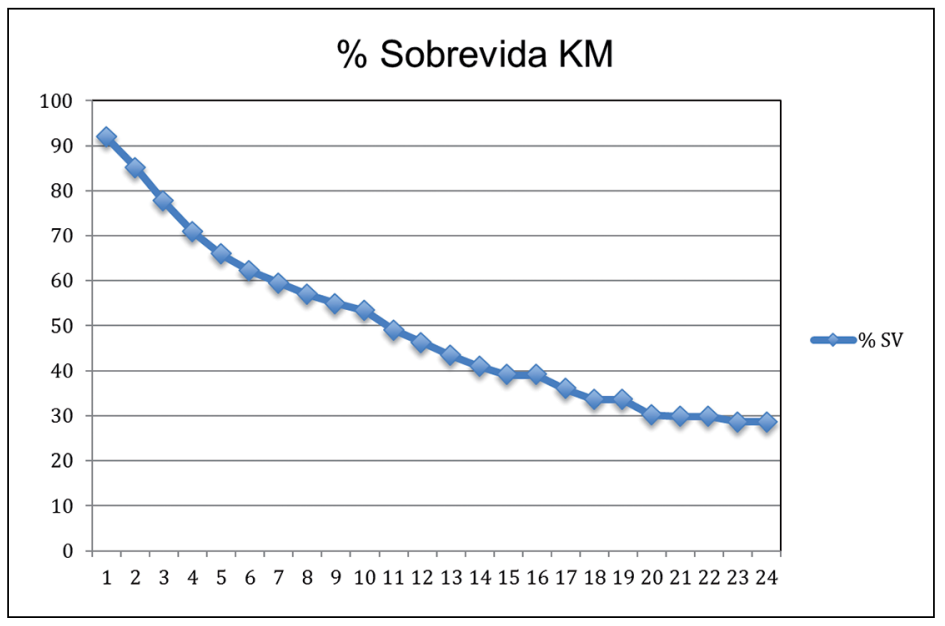

Figura 1. Curva Kaplan-Meier de sobrevida en meses. 
Se reportó una sola complicación en un paciente tratado con 8 Gy en 1 fracción, quien sufrió una fractura de fémur.

\section{Discusión}

El manejo paliativo de los pacientes con cáncer representa prácticamente la mitad de las indicaciones de radioterapia ${ }^{3,8}$. En esta revisión se analizaron 197 pacientes que ingresaron para manejo de dolor por metástasis óseas. Se observó una mediana de tiempo entre el inicio de los síntomas y el inicio de la radioterapia de 4 meses, un período largo considerando que se trata de pacientes con enfermedad avanzada, en su mayoría con expectativa de vida limitada. Este problema también ha sido reportado por otros investigadores, observando que las causas de retardo pueden ser imputables al Centro de Radioterapia. Es así que el estudio de Barnes et $\mathrm{al}^{9}$, muestra que dentro de los factores que los médicos familiares consideran como barreras para acceder a radioterapia paliativa, están el retraso en obtener una cita con el radioterapeuta, retraso en el inicio de la terapia después de la primera consulta con el especialista y dificultades en el transporte y alojamiento del paciente. Esto último puede ser más importante en pacientes de regiones que se encuentran más lejos del centro de radioterapia.

Hay además otros factores que se agregan al retardo en la derivación. Existe un desconocimiento de las indicaciones de radioterapia en pacientes con cáncer diseminado. En este mismo estudio, un $87 \%$ de los médicos encuestados declaró no estar cómodo con el conocimiento que tenían sobre la radioterapia paliativa. Un $84 \%$ declaró pensar que el tiempo medio de respuesta al tratamiento era menos de 4 semanas $^{9} \mathrm{y}$, aunque en este período de tiempo sí puede alcanzarse algún grado de alivio del dolor, existen reportes que indican que el alivio completo se alcanza en sólo un $25 \%$ de los pacientes al mes de radioterapia y un $41 \%$ de los pacientes presenta alivio del dolor de al menos $50 \%$ al mes de tratados ${ }^{10}$. Es probable que esto determine que los médicos familiares consideren que la radioterapia paliativa no es efectiva al no evidenciar alivio del dolor en el tiempo que ellos estipulan adecuado. Es así, como estos factores puedan explicar la reticencia a la derivación rápida del paciente con dolor a radioterapia.

Si bien la mayoría de nuestros pacientes recibieron el esquema de tratamiento de 8 Gy en una fracción, hubo un $40 \%$ de distintos tratamientos en que se usaron múltiples fracciones. Como se mencionó anteriormente, existen diversos estudios que muestran que el tratamiento con una sola dosis es igualmente efectivo que los tratamientos prolongados. En un meta análisis ${ }^{4}$ se mostró una tasa de respuesta global de 58\% para los pacientes tratados con una sola dosis y $59 \%$ para aquellos tratados con múltiples fracciones. La respuesta completa fue de $23 \%$ y $24 \%$ para los que recibieron una y múltiples fracciones respectivamente, sin diferencias estadísticamente significativas en la tasa de respuesta. En otro estudio ${ }^{6}$, se demostró lo mismo con cifras similares, respuesta completa de $13 \%$ y respuesta parcial de $52 \%$ a las 12 semanas para el grupo que recibió 8 Gy, y respuesta completa y parcial de $11 \%$ y $51 \%$ respectivamente a las 12 semanas para el grupo que recibió $30 \mathrm{~Gy}$.

En otro estudio que comparó 8 Gy en 1 fracción con 30 Gy en 10 fracciones en el manejo de metástasis óseas vertebrales ${ }^{11}$, también se vio que ambos esquemas eran equivalentes en el alivio del dolor y en el requerimiento de uso de narcóticos para el alivio del dolor a 3 meses, observándose menor toxicidad en grupo de $8 \mathrm{~Gy}$, aunque mayor tasa de retratamiento, $15 \%$ versus $5 \%$. Así también, al analizar distintos esquemas de tratamiento en pacientes con compresión medular por metástasis, no hubo diferencias en la presencia y duración de la respuesta, sobrevida o toxicidad entre aquellos que recibieron un esquema corto de 8 Gy en 2 fracciones versus aquellos con un esquema dividido de $5 \mathrm{~Gy} x$ 3 fracciones y 3 Gy x 5 fracciones ${ }^{12}$.

En nuestro estudio la tasa de re irradiación fue de $5 \%$, la mayoría en los pacientes que fueron tratados con una sola fracción de $8 \mathrm{~Gy}$. Esto concuerda con lo reportado en la literatura ${ }^{4}$, donde se describe una probabilidad de retratamiento 2,5 veces mayor en el grupo de mono dosis que en multi fracción. Otro estudio $^{6}$, muestra una gran diferencia entre ambas ramas, donde un $28 \%$ de los pacientes que recibieron una sola dosis fueron reirradiados en comparación a un $2 \%$ de los que recibieron $30 \mathrm{~Gy}$. El estudio de Hartsell ${ }^{7}$, también reporta grandes diferencias, con un $18 \%$ de re-irradiación en el grupo de 8 Gy y $9 \%$ en el grupo de $30 \mathrm{~Gy}$.

Por otro lado, en un estudio publicado recientemente $^{13}$, se evaluó la respuesta en términos de alivio del dolor a 2 meses en pacientes reirradiados por metástasis óseas. Se comparó 8 Gy en 1 fracción con 20 Gy en varias fracciones. No hubo diferencia en respuesta, en libertad de progresión de síntomas, ni alteración de actividades de vida diaria. Un 34\% y $35 \%$ de los pacientes presentaron mejoría de síntomas entre los que recibieron 8 Gy y 20 Gy respectivamente $(\mathrm{p}=0,87)$. Tampoco se observó diferencia en sobrevida global, siendo 9,3 meses y 9,7 meses $(p=0,66)$ para 8 y 20 Gy respectivamente. No hubo diferencia en respuesta de acuerdo al esquema de tratamiento anterior recibido. La modalidad de una sola fracción se asoció a menos efectos adversos.

Si bien la tasa de retratamiento reportada en nues- 
tra revisión es baja, cabe señalar que no se contó con la totalidad de los datos, por lo que puede estar subestimada.

La decisión de reirradiación habitualmente queda a criterio del médico tratante. Puede existir una inclinación a no indicar más tratamiento con irradiación a pacientes que ya han recibido dosis mayores (20-30 Gy), esto produce un sesgo hacia el retratamiento. En esta misma línea, sí se aplicó mono dosis, existe mayor posibilidad de indicar retratamiento ya que no se teme a toxicidad tardía. Además, los esquemas de multi fracción han demostrado mayor toxicidad aguda ${ }^{7}$, por lo que es menos probable que se indique retratamiento en un paciente que toleró mal el primer curso de terapia.

En este trabajo se reportó una sola complicación severa. En la literatura, tampoco se reporta diferencia en la incidencia de complicaciones como fractura patológica o compresión medular ${ }^{4,7,13}$.

La mayoría de los pacientes que reciben tratamiento paliativo tienen una expectativa de vida limitada, en nuestra serie la mediana de sobrevida fue de 10,5 meses. Esto es similar a lo observado en otras series, donde la sobrevida media para dos grupos de tratamiento fue de 9,1 meses en la rama de 8 Gy y 9,5 meses en la rama de 30 Gy de tratamiento 6 . Así también, la sobrevida a 12 y 24 meses de $46 \%$ y $29 \%$ similar a lo reportado en la literatura para distintas ramas de tratamiento, con una sobrevida global de $41 \%$ y $22 \%$ al año y dos años de seguimiento para los pacientes que recibieron $8 \mathrm{~Gy}$, y $42 \%$ y $22 \%$ al año y dos años para los pacientes tratados con $30 \mathrm{~Gy}^{7}$. Estas cifras recalcan la importancia de acortar la duración de los tratamientos en pacientes que tienen expectativas de vida corta. Esto permite mejorar la calidad de vida del paciente y lo que también es muy importante, la de su familia. Sin duda que la medición de sobrevida no es una medición objetiva del efecto de un tratamiento paliativo, de aquí que lo importante sea la evaluación del efecto perseguido, es decir, el alivio del síntoma que produce el deterioro en la calidad de vida: el dolor.

\section{Conclusión}

Dado que el alivio del dolor es similar entre modalidades terapéuticas prolongadas en el tiempo y aquellas en que se usa mono dosis, la sobrevida no se altera y no hay mayor incidencia de complicaciones, la indicación de un tratamiento paliativo más corto, de más fácil acceso para el paciente y de menor $\operatorname{costo}^{10}$ parece ser la alternativa más razonable. Así también, una correcta evaluación del paciente antes de iniciar la terapia para estimar de la manera más precisa posible su condición y expectativa de vida, puede evitar sobretratamiento y brindar al paciente la mejor calidad de vida posible. Recordemos que especialmente para el paciente portador de un cáncer avanzado el tiempo que importa más es aquel sin síntomas y sin doctor.

\section{Referencias}

1. Hoeler D. Radiotherapy for palliation of symptoms in incurable cancer. Curr Probl Cancer 1997;21:129-83.

2. Oldenburg $\mathrm{N}$. The role of palliative radiation in the management of brain, spinal cord, and bone metastases. Med Health R I. 2006;89:59-62.

3. Ashby M. The role of radiotherapy in palliative care. $\mathrm{J}$ Pain Symptom Manage 1991;6:380-8.

4. Chow E, Harris K, Fan G, Tsao M, Szef W. Palliative radiotherapy trials for bone metastases: A systematic review. J Clin Oncol. 2007;25:1423-36.

5. Gojkovic A, Kovac V, Strojan P. Radiotherapy in palliative treatment of painful bone metastases. Radiol Oncol. 2009;43:213-24.

6. Foro P, Valls A, Carles J, Lynd F, Sanz X, Rodríguez N, et al. Randomized clinical trial with two palliative radiotherapy regimens in painful bone metastases: 30 Gy in 10 fractions compared with 8 Gy in single fraction. Radiotherapy and Oncology 2008;89:150-5.

7. Hartsell W, Scott C, Watkins D, Scarantino C, Ivker $\mathrm{R}$, Roach $\mathrm{M}$, et al. Randomized trial of short versus long-course radiotherapy for palliation of painful bone metastases. J Natl Cancer Inst 2005;97:798-804.

8. Gripp S, Mjartan S, Boelke E, Willers R. Palliative radiotherapy tailored to life expectancy in end-stage cancer patiens. Reality of myth? Cancer 2012;116:32516.

9. Barnes E, Parliament H, Hanson J, Watanabe S, Palmer J, Bruera E. Palliative radiotherapy for patients with bone metastases: survey of primary care physicians. Radiotherapy and oncology 2003;67:221-3.

10. Vakaet L, Boterberg T. Pain control by ionizing radiation of bone metastasis. Int J Dev Biol. 2004;48:599606.

11. Howell D, James J, Hartsell W, Suntharalingam M, Machtay M, Suh J, et al. Randomized trial of shortcourse versus long-course radiotherapy for palliation of painful vertebral bone metastases: A retrospective analysis of RTOG 97-14. J Clin Oncol. 2009;27:15s.

12. Maranzano E, Bellavita R, Rossi R, De Angelis V, Frattegiani A, Bagnoli R, et al. Short-course versus split-course radiotherapy in metastatic spinal cord compression: results of a phase III, randomized, multicenter trial. J Clin Oncol. 2005;23:3358-65.

13. Chow E, van der Linden Y, Roos D, Hartsell W, Hoskin $\mathrm{P}, \mathrm{Wu}$ J, et al. Single versus multiple fractions of repeat radiation for painful bone metastases: a randomized, controlled, non-inferiority trial. Lancet Oncol. 2014;15:164-71. 